\title{
A Abordagem Cognitivo-Comportamental dos Sonhos de Alcoolistas
}

\author{
Renata Brasil Araujo* \\ Margareth da Silva Oliveira** \\ Luciane Benvegnu Piccoloto*** \\ Mylène Magrinelli**** \\ Karen Szupszynski ${ }^{* * * * *}$
}

\section{INTRODUÇÃO}

A dependência de uma substância química como o álcool é um diagnóstico freqüentemente encontrado na clínica, sendo fundamental a compreensão de suas implicações para que se possa lidar, de uma forma mais efetiva, com este problema. ${ }^{1}$

São várias as modalidades de tratamento disponíveis para este tipo de transtorno mental, porém sabe-se que a abordagem de impacto mais indicada para o alcoolismo é a Entrevista Motivacional e a Terapia Cognitivo-Comportamental, havendo uma ênfase para a Prevenção da Recaída². Assim, alguns instrumentos terapêuticos amplamente utilizados na Terapia Psicanalítica, como por exemplo, os sonhos, tiveram de ser estudados sob este enfoque, para que pudessem fazer parte do arsenal terapêutico dos profissionais da área da dependência química ${ }^{3-6}$.

\footnotetext{
* Doutoranda em Psicologia - PUCRS.

** Doutora em Ciências - UNIFESP.

*** Mestranda em Psicologia Clínica - PUCRS.

${ }^{* * * *}$ Doutoranda em psiquiatria - UFRGS.

${ }^{* * * *}$ Auxiliar de Pesquisa e Bolsista CNPq.
}

Segundo Martinez ${ }^{7}$, o ser humano, apesar de toda a evolução do conhecimento, ainda não tem respostas definitivas quanto aos processos que conduzem o sono e os sonhos. Existem, no entanto, várias teorias a respeito do sono, como as que consideram ser sua função a conservação de energia e a restauração de processos químicos e físicos deteriorados na fase de vigília.

\section{OBJETIVO}

O presente trabalho tem como objetivo realizar uma revisão teórica a respeito do sono e dos sonhos de alcoolistas de acordo com a Teoria Cognitivo-Comportamental, aprofundando aspectos cognitivos e neurológicos.

\section{MÉTODO}

Foi realizada uma pesquisa no Banco de dados Psyclnfo, de artigos indexados, publicados entre os anos de 1997 e 2002. Os descritores utilizados foram: alcohol, dream, sleep e cognitive.

Devido a existência de poucos estudos 
dentro da abordagem pesquisada, foram feitos contatos, via e-mail, com os Doutores Cláudio Colace $^{14}$ e Michael Shredl ${ }^{12,17}$ que indicaram outras leituras.

Além disto, foram pesquisados artigos ${ }^{5,6,11}$, livros $^{1,2,4,7,9,15}$ e uma Dissertação de Mestrado ${ }^{18}$ escritos no Brasil para incrementar a revisão.

\section{ESTÁGIOS DO SONO}

O processo do sonho é distinto do sono, pois, neste primeiro, são produzidas descargas elétricas, imagens, sons e sensações, ocorrendo um característico movimento ocular. Esta fase do sono foi denominada, devido a este movimento dos olhos, Fase REM (Rapid eye movements) ou, devido ao contraste da ativação cerebral desta fase com o relativo "descanso" do cérebro quando se dorme, de Sono Paradoxal. A Fase REM é considerada tão importante que o restante do sono é denominado Não-REM ${ }^{7}$. Em laboratório, pessoas privadas do Sono REM podem, eventualmente, segundo pesquisas ${ }^{8}$, apresentar irritabilidade, ansiedade e comportamento psicótico.

Os vários estados do sono com seus específicos estágios puderam ser observadas por meio de um registro chamado polissonografia e estão subdivididos, de acordo com Martinez ${ }^{7}$ e Reimão ${ }^{9}$, da seguinte forma:

\section{Estado de vigília:}

Ocorre quando se está acordado e quando ocorrem despertares breves durante o sono (costumam ocorrer de 5 a 15 vezes por hora), sendo comum serem acompanhados de movimentos corporais (como virar-se na cama). Esta fase representa $15 \%$ do tempo total de sono e caracteriza-se por dois tipos de ondas cerebrais: alfa, quando se fecha os olhos; e beta, uma vigília atenta que ocorre, por exemplo, quando se realizam cálculos mentais.

\section{Sono Não-REM:}

Estágio 1 - é a transição da vigília para o sono e dura de segundos até 3 minutos. Caracteriza-se por ondas teta de baixa voltagem e freqüência mista e corresponde a uma porção de 5 a $10 \%$ do sono. É considerado um semisono, pois se o indivíduo for despertado neste estágio, conseguirá lembrar de fatos ocorridos no ambiente.

Estágio 2 - Ocorre após o Estágio 1 e é caracterizado por dois tipos de onda: os complexos $K$ e os fusos do sono, cada um deles durando 2 segundos e ocorrendo cerca de 3 vezes por minuto. Corresponde a um intervalo entre 45 e $55 \%$ do sono total. Este estágio é considerado o verdadeiro início do Sono Não REM, pois é mais difícil acordar a pessoa neste estágio e há um maior distanciamento com relação aos fatos ocorridos no meio ambiente.

Estágios 3 e 4 - Depois de um período aproximado entre 15 e 20 minutos de sono, podem ser verificadas ondas delta, lentas e amplas. Há, neste estágio, uma diminuição da pressão arterial e freqüência cardíaca, ficando a respiração irregular. Esta etapa corresponde a $30 \%$ do sono em crianças, diminuindo esta proporção com a idade. Nesta fase, despertar um indivíduo pode demorar até 20 minutos.

\section{Sono REM:}

Nesta fase ocorre um relaxamento muscular de quase todo o corpo, sendo as únicas exceções o diafragma e o músculo dos olhos. Os movimentos oculares rápidos ocorrem em intervalos irregulares, ocupando $10 \%$ de toda a fase REM. As freqüências cardíaca e respiratória, assim como a pressão arterial, tornam-se variáveis, podendo ocorrer episódios de paradas respiratórias. A fase REM costuma demorar de 65 a 120 minutos para aparecer em seu primeiro período após adormecer. Estes períodos de sono REM se repetirão a cada 90 minutos e sua duração será progressiva.

Apesar de existirem muitas diferenças entre o sono e o período em que estamos acordados, em vigília, pesquisas sugerem que o nível de ativação dos neurônios reticulares na porção central do cérebro de um indivíduo dormindo e o de um acordado são idênticos; assim como há uma equivalência, em ambos, nas funções do sistema tálamo-cortical ${ }^{8}$.

\section{OS SONHOS}

Os sonhos ocorrem predominantemente na Fase REM, e durante o seu aparecimento, são produzidas descargas que podem atingir diversas áreas cerebrais, o que dará a "tonalidade" emocional do sonho. Assim, um sonho pode causar medo ou riso, sem que seu conteúdo emocional esteja associado a estas respostas. É interessante destacar que nem sempre se consegue lembrar dos sonhos, pois estas recordações necessitam de que ocorra um despertar prolongado durante o sono, já que a memória só funciona depois de 5 minutos de vigília ${ }^{7}$.

Freud $^{10}$ refere que estímulos sensoriais ex- 
ternos e internos podem alterar o conteúdo de sonhos e, no início de suas pesquisas nesta área, antes de se aprofundar nas relações com os impulsos inconscientes (desejos), utilizou o método científico-experimental para avaliar essas influências de ordem fisiológica.

O sonho, mesmo tendo esta forte influência de ordem neurológica, não se pode esquecer, tem uma importante função adaptativa, à medida que apresenta situações já vividas e possibilita um preparo para que os mesmos erros não sejam cometidos. Sob este ponto de vista, haveria dois tipos de sonhos: "os moduladores" e os "formadores de estratégias". Os "moduladores" são aqueles que apresentam situações nas quais são utilizadas estratégias anteriormente formuladas em vigília e que precisam de reforço ou inibição; e os "formuladores de estratégias" são os sonhos que possibilitam que sejam criadas estratégias cognitivas para capacitar o indivíduo que sonha para lidar com experiências novas, para as quais não havia previamente se preparado. Isto ocorre independente do indivíduo recordar o conteúdo de seus sonhos, pois, no sonho, as emoções formam um núcleo afetivo em torno do qual são feitas formulações cognitivas, havendo uma rede de associação subliminar entre afeto e cognição, que pode ser resgatada quando do surgimento de uma situação que está relacionada ao conteúdo onírico em questão ${ }^{11}$.

\section{PESQUISAS RELACIONADAS AOS SONHOS}

Chaves, Caixeta e Machado ${ }^{11}$ fizeram uma revisão das teorias a respeito dos sonhos, utilizando como suporte a análise do caso de 5 pessoas, adultas e hígidas monitoradas em um laboratório do sono. Estes indivíduos foram despertados, em dias separados, tanto durante a fase REM, quanto durante o REM rápido, sendo este último, na maioria das vezes, próximo da transição REM - Não REM (Estágio 1). Os pacientes acordados no decorrer do REM mais rápido referiram sonhos mais lógicos em seu conteúdo, com uma maior elaboração cognitiva e apresentaram um desejo de acordar nos momentos ansiogênicos dos sonhos. Em contrapartida, aqueles despertados durante o REM mais lento descreviam sonhos "bizarros", sem uma elaboração cognitiva e não havendo uma crítica a respeito de estar sonhando ou o desejo de controlar o sonho.

Chaves et al. ${ }^{11}$ sugerem ainda que a mudança do padrão do sonho - do mais emocional ao mais lógico - está relacionada a uma oscilação de dominância límbica e frontal em cada período do sono REM, estando os sonhos mais coerentes presentes no período de atividade eletroencefalográfica frontal mais rápida e os bizarros, em um período de menor ativação frontal, na qual haveria uma dominância límbica. No início do sono REM, há uma predominância dos inputs límbicos sobre os outputs frontais, ocorrendo, em decorrência disto, impressões afetivas puras, sem que haja uma verificação frontal efetiva a ponto de tornar coerente o conteúdo onírico de indivíduos despertados nesta fase.

A partir da leitura de alguns trabalhos que demonstraram que situações de estresse e preocupações da vida diária são refletidas no conteúdo dos sonhos, Schredl ${ }^{12}$ decidiu pesquisar a freqüência com que atividades com uma reduzida intensidade emocional - como usar o computador, ler e escrever - estavam presentes no conteúdo dos sonhos. Em seus achados, concluiu que estes conteúdos não eram freqüentes nos sonhos da amostra estudada $(\underline{N}=60)$, o que poderia estar associado ao fato que, durante o sono REM, há uma forte ativação do sistema límbico, estando a área frontal relacionada ao pensamento racional, menos ativada neste período do que no estado de vigília.

\section{OS SONHOS DE DEPENDENTES QUÍMICOS}

Kalra, Natu, Deswal e Agarwal ${ }^{13}$ realizaram uma pesquisa com pacientes psiquiátricos, entre eles dependentes químicos $(28 \%)$, que estavam utilizando psicofármacos, como benzodiazepínicos, antidepressivos, antipsicóticos e moderadores de humor, durante o período de internação hospitalar, sendo analisado como eram os sonhos desta amostra $(\underline{N}=50)$. Os sonhos destes pacientes foram comparados aos de voluntários normais, obtendo-se os seguintes resultados: os pacientes recordaram menos os seus sonhos, seus conteúdos foram mais aterrorizantes e ocorreram mais repetições dos relatos, quando comparados com o grupo controle. Estas diferenças ocorreram mesmo antes do aparecimento da doença mental. Após o início da farmacoterapia, $78 \%$ dos pacientes observados tiveram seus sonhos suprimidos, sendo que, do grupo que tomava benzodiazepínicos, apenas $18 \%$ não reduziu seus sonhos. Esta redução significativa associada aos benzodiazepínicos pode ser explicada pela sua ação diminuindo o Sono REM e o Estágio 4 do sono, fases estas relacionadas à produção do sonho.

Não é possível afirmar, porém, de acordo com Kalra ${ }^{13}$, que se os sonhos são suprimidos 
com o uso deste tipo de medicação ou se a sua ação causa apenas um prejuízo na memória onírica.

Pesquisa, realizada por Colace ${ }^{14}$, com sonhos de pacientes dependentes de heroína, durante a fase inicial de abstinência, em um tratamento de substituição por metadona, utilizou uma amostra de quatro pacientes com seus respectivos relatos de seus conteúdos oníricos e observou ser freqüente o uso da heroína nos sonhos destes sujeitos, sendo este sucedido por sentimentos de ansiedade e culpa, que muitas vezes causavam o seu despertar. Este sonhar com o uso da substância, em seus achados, demonstrou ser mais comum na fase inicial da abstinência, quando há um aumento no craving e a presença de sintomas físicos e psicológicos de privação, diminuindo na medida em que o craving diminuía na fase de vigília. Um dos pacientes desta amostra referiu que este tipo de sonho, quando da diminuição do craving, poderia não diminuir, mas sim ser modificado, passando o "autor" de um papel de protagonista a mero espectador.

Segundo Kalra ${ }^{13}$, se estudos em laboratórios do sono, nos quais sejam avaliadas as modificações nas diferentes fases do ciclo do sono ocasionadas por psicofármacos, forem associados à análise dos sentimentos subjetivos motivados pelo conteúdo dos sonhos, será possível um aprofundamento na díade droga/sonho.

O álcool que, durante o seu uso, pode, a princípio, prolongar o sono e reduzir a quantidade de sono REM, quando é retirado, tem um efeito contrário: o sono é diminuído, a ansiedade aumenta e ocorrem muito mais sonhos pelo aumento compensatório do Sono REM ${ }^{15}$.

O sono de alcoolistas, na fase de desintoxicação, segundo alguns autores ${ }^{16}$ tem uma menor duração, é mais fragmentado e superficial no início da abstinência, melhorando lentamente no decorrer do primeiro ano, caso não ocorram recaídas. Algumas pessoas, no entanto, segundo estes achados, permaneceram com alteração no sono mesmo após 27 meses de abstinência. Insônia e fragmentação do sono poderiam estar associadas a uma recaída em uma fase de quatorze meses de abstinência, porém este tipo de relação ainda não está clara, merecendo novos estudos.

Ao investigar 74 pacientes alcoolistas no período de 2 a 4 semanas após a retirada do álcool, Schredl17 constatou que a recordação dos sonhos por este grupo, tanto na fase de desintoxicação, como durante o uso do álcool, está levemente aumentada, o que poderia ser explicado por um aumento na freqüência de despertares noturnos- o despertar durante ou após a fase REM parece ocasionar o armazenamento do conteúdo sonhado na memória recente, o que facilitaria o seu resgate. A presença de pesadelos, é preciso salientar, foi comum nesta amostra.

A ocorrência de apnéia do sono, bem como mioclonia, também parecem estar associadas a este incremento na capacidade de lembrança dos conteúdos oníricos, nestes indivíduos. Nesta mesma pesquisa, Schred ${ }^{17}$ observou que fatores como: dias de abstinência, duração do alcoolismo e a quantidade de álcool ingerida diariamente não foram relacionadas à freqüência da recordação dos sonhos, havendo, no entanto, uma correlação positiva com quantidade de sono REM, latência curta REM e uma elevada densidade do sono REM. Um estado negativo antes de dormir, nesta pesquisa, esteve associado a uma maior prevalência de emoções negativas no conteúdo dos sonhos. Deste estudo, constou uma fase de follow up, na qual, após 1 ano, foram comparados pacientes que recaíram (29) e que ficaram abstinentes (19) e não foram encontradas diferenças significativas quanto à freqüência da recordação dos sonhos nos dois grupos $(p=0,2530)$, porém os pacientes que permaneceram abstinentes demonstraram uma maior preocupação com o conteúdo de seus sonhos durante o período de vigília $(\mathrm{p}=0,0315)$.

Foi pesquisada por Araujo $^{18}$, em seu estudo com alcoolistas no período de desintoxicação ( $n=77)$, a associação entre apresentar despertares noturnos durante o sono e a produção de sonho, o que não pode ser demonstrado $(\underline{p}=0,2341)$. No entanto, ao analisar esta característica do sono com o sonhar com o álcool, a autora obteve uma associação significativa $(p=0,0000)$ : os pacientes que apresentavam um aumento nos despertares noturnos relatavam mais este tipo de sonho.

À medida em que $100 \%$ dos sonhos são compostos de imagens visuais - e 75\% têm elementos auditivos ${ }^{9}$, bem como seus conteúdos têm, como já foi descrito, uma "função adaptativa"11 -, o material onírico pode ser utilizado como mais um instrumento para avaliar o craving em indivíduos alcoolistas ${ }^{19}$ e para que sejam elaboradas estratégias de prevenção de recaída, sendo útil que se ampliem pesquisas com este enfoque na área da dependência química ${ }^{17}$.

Choi ${ }^{20}$ observou que pacientes em fase de desintoxicação do álcool que sonham estar bebendo apresentam menos ansiedade e têm 
maiores condições para se manterem abstinentes por um maior período de tempo do que aqueles que não têm este tipo de sonhos, pois este primeiro grupo teve, mesmo que de uma forma parcial, a satisfação de seu desejo. Outros autores ${ }^{21}$, no entanto, pesquisando pacientes com dependência de múltiplas drogas (incluindo o álcool), encontraram achados que apontam na direção contrária: sonhar com o uso da substância química é um prognóstico desfavorável à manutenção da abstinência. Christo e Franey ${ }^{21}$ relatam ainda que $84 \%$, destes 101 sujeitos pesquisados comentaram ter sonhado com substâncias psicoativas, o que ocorreu com maior freqüência quando estavam abstinentes. Sonhar com estas substâncias, segundo esta pesquisa, está associado com a ocorrência de craving e insônia. Johnson ${ }^{22}$, quando acompanhou pacientes dependentes do álcool, em follow-up, após alta da internação hospitalar, constatou não haver uma relação entre recaída e o ato de embriagar-se nos sonhos, porém concluiu da importância de que sejam feitas novas pesquisas nesta área, já que sonhar com a bebida alcoólica é, segundo seus achados, mais freqüente em pacientes alcoolistas do que em um grupo controle que não tem este tipo de comportamento-problema.

Araujo ${ }^{18}$, em sua pesquisa com pacientes alcoolistas $(n=77)$ nos três primeiros dias de desintoxicação, observou que a maioria não apresentou o comportamento de sonhar com álcool $(72,7 \% ; n=56)$ neste período, porém este tipo de sonho estava associado com um aumento no craving ( $\mathrm{p}=0,000)$. A média das notas do craving no grupo que sonhou com álcool foi igual a $18,38(\underline{S D}=10,45)$, enquanto no que não sonhou foi $8,08(\underline{S D}=8,21)$. Peters ${ }^{23}$, por outro lado, demonstrou haver uma diferença significativa entre homens alcoolistas e não-alcoolistas quanto ao conteúdo de seus sonhos: os primeiros, ao contrário dos demais, costumam sonhar com a bebida alcoólica e com a transição para a sobriedade.

À medida em que os alcoolistas utilizam seus sonhos para expressar o craving, tornam possível que seja trabalhada a prevenção à recaída em seus tratamentos psicoterápicos ${ }^{18,23}$.

Do ponto de vista teórico-metodológico do estudo dos sonhos, o acompanhamento de indivíduos dependentes químicos é uma oportunidade para se examinar o papel da necessidade/desejo na formação dos sonhos, pois nestes casos, comparativamente com sujeitos que não apresentam esta psicopatologia, esta variável (necessidade/desejo) está presente em uma lada, na medida em que se pode ter o controle do tempo de abstinência ${ }^{14}$.

\section{OS SONHOS SOB O ENFOQUE COMPORTAMENTAL}

Apesar de estudos consistentes sobre sonhos terem iniciado há mais de cem anos com Freud $^{10}$, só recentemente os profissionais têm procurado aprimorar o seu conhecimento neste enfoque. Os adeptos da Teoria Comportamental têm poucos, porém bem atualizados, estudos de sonhos, apesar de este ser um tema muitas vezes referido pelos pacientes na clíni$\mathrm{ca}^{3-6}$.

Segundo Delitti ${ }^{6}$, o sonho, a partir de sua análise funcional, é um instrumento através do qual o terapeuta poderá ter acesso à história do cliente e criar condições para que, no ambiente terapêutico, sejam evocadas as respostas emocionais do mesmo, as quais, neste contexto, podem mais facilmente ser modificadas. Guillar$\mathrm{di}^{4}$ considera que sonhar é comportar-se e, portanto, a interpretação dos sonhos deve integrar seus conteúdos com outros comportamentos emitidos pelo indivíduo em questão.

Para Skinner ${ }^{3}$, sonhar é uma resposta perceptual que pode ocorrer na falta de estímulos externos, em função de que aprendemos a nos comportar de acordo com o que fomos ou seremos reforçados. Assim, os sonhos podem funcionar como estímulos abstratos para os quais se produzirá uma resposta, dependendo da história e dos reforços que marcaram o indivíduo.

Obviamente, existem motivos para determinados comportamentos serem relatados na clínica, através de sonhos e não de relatos verbais diretos: a linguagem metafórica dos sonhos facilita a expressão de sentimentos e pensamentos que seriam rechaçados, ou mesmo punidos, se fossem verbalizados de outra forma, ou em outro contexto. Isto não significa que o indivíduo escolhe conscientemente um jeito de se comunicar com seu terapeuta: o comportamento de sonhar é regido pelos mesmos mecanismos de outras categorias de comportamentos, nas quais, muitas vezes, não se conhece o que motivou um determinado comportamento 4 .

Na prática terapêutica, Delitti ${ }^{6}$ relata que a análise dos sonhos deve partir de um questionamento para o cliente a respeito do que ele entendeu daquilo que sonhou e, de acordo com a sua resposta, identificar associações funcionais com outros padrões de sua vida. Guillardi ${ }^{4}$ salienta que, após a intervenção, o terapeuta deve estar atento aos resultados para que as 
hipóteses levantadas no decorrer deste processo possam ser confirmadas ou refutadas. Guillardi ${ }^{4}$ argumenta, ainda, que a aura de mistério que envolve os sonhos cria um plus motivacional, o qual poderia ter uma maior influência no tratamento de alguns clientes. No entanto, é preciso lembrar que isto precisaria ser pesquisado de uma forma mais consistente.

Delitti ${ }^{6}$ comenta que o relato dos sonhos no ambiente terapêutico é um comportamento que pode ou não ser reforçado pelo terapeuta, porém não deve, de forma alguma, ser ignorado, e sim compreendido funcionalmente como outros eventos verbais. Além disto, esta análise dos sonhos com o cliente fortalece o vínculo terapêutico: a atitude empática e interessada do terapeuta reforça positivamente a emissão do comportamento verbal de seu cliente, levandoo, segundo Guillardi ${ }^{4}$, a crescer no aspecto referente a seu autoconhecimento.

\section{DISCUSSÃO}

Depois que se estudam os mecanismos que produzem os sonhos, não se consegue mais analisá-los dissociadamente do período do sono correspondente aos mesmos ${ }^{7,9,8,11-}$ 13,15,17, ficando muito difícil ignorar esta ligação no dia a dia da clínica psicológica e, mais especificamente, na área da dependência química.

Os estudos ${ }^{7-18,21}$ apontam que alterações no estado fisiológico e no sono dos indivíduos modificam significativamente a produção e o conteúdo de seus sonhos, o que pode ser observado, na nossa experiência como terapeutas que acompanham a desintoxicação de dependentes químicos, pela dificuldade de alguns destes de recordarem seus sonhos e pelo aumento na produção onírica daqueles que têm aumentado o número de despertares noturnos.

Conhecer a respeito das alterações no sono de alcoolistas pode servir como alerta quanto a um risco de recaída, já que alguns trabalhos apontam neste sentido ${ }^{18}$. Isto, sem dúvida alguma, indica que a avaliação do sono não pode ser desconsiderada pelos profissionais, durante o tratamento de dependentes químicos, tanto na fase em que estes pacientes estão fazendo uso da substância psicoativa, quanto na fase de desintoxicação.

Muitos pacientes relatam que o uso da bebida alcoólica serve como um auxílio para dormir, no entanto, não desconsiderando o caráter sedativo do álcool, observa-se que a qualidade do sono, tanto durante o consumo desta substância, como da fase de desintoxicação, está prejudicada, o que também está de acordo com a literatura pesquisada ${ }^{13,15-18}$. Portanto, informar o alcoolista quanto a esta distorção cognitiva serve para motivá-lo para que aja no sentido de conseguir e manter a abstinência desta substância.

Pode ser útil, no decorrer do tratamento, solicitar ao dependente que tem esta crença na importância do efeito sedativo da bebida alcoólica como auxiliar no sono, que faça um registro diário de seu sono, explicitando a hora em que deitou, na qual conseguiu dormir, o número de despertares noturnos, a hora em que acordou, se conseguiu recordar seus sonhos, bem como a sua opinião quanto à qualidade de cada noite dormida. Um outro instrumento que pode ser utilizado nesta avaliação é o Questionário do Sono e dos Sonhos elaborado por Araujo ${ }^{18}$. Estes dados devem ser discutidos com o paciente para que ele possa perceber que o álcool, de fato, não o ajuda a ter um sono satisfatório.

Percebe-se na prática clínica, assim como através de pesquisas ${ }^{18}$, que os dependentes químicos, muitas vezes, trazem às consultas relatos de sonhos - alguns relacionados ao uso de substâncias psicoativas - e que os terapeutas acabam não sabendo o que fazer com estas informações, deixando os pacientes desapontados.

Como o tratamento na área da dependência química deve ser focal e o trabalho dos aspectos psicodinâmicos, sendo ansiogênico, é contra-indicado para esta clientela - pelo menos em um primeiro momento ${ }^{2}-$, os sonhos que estão muito identificados com teoria Psicanálítica $^{10}$ são vistos de forma preconceituosa pelos profissionais especializados nesta patologia.

O relato dos sonhos, no entanto, pode ser um instrumento terapêutico muito valioso por oferecer pistas, por exemplo, de acordo com algumas pesquisas ${ }^{14,18}$, a respeito do quanto 0 craving está presente em dependentes químicos que estão tentando manter-se em abstinência ou que pretendem interromper o uso de uma droga. À medida em que sonhar com a bebida alcoólica pode estar associado a um aumento no craving $^{14,18}$, a identificação precoce deste tipo de sonho como uma situação de risco poderá trazer resultados mais efetivos quanto à prevenção da recaída.

O sonhar com a bebida alcoólica, segundo Araujo ${ }^{18}$, não é tão freqüente, porém acreditamos que este comportamento ocorre, às vezes, sem que seja relatado ao terapeuta ou questionado por este, perdendo-se uma oportunidade de avaliar o desejo do indivíduo de beber. 
Quanto ao fato de sonhar com álcool servir como uma forma de proteção para uma futura recaída, os estudos não são conclusivos ${ }^{20-22}$. Pensamos serem necessárias novas pesquisas para que este ponto seja melhor esclarecido.

Vários autores encaram o sonho como uma possibilidade de se aprender mais a respeito dos pacientes, de seus comportamentos e de sua história ${ }^{3-6}$, e outros percebem que estes funcionam até mesmo como "moduladores" e "formadores de estratégias adaptativas"11, além de serem úteis no fortalecimento do vínculo terapêutico ${ }^{6}$.

Com certeza, os pacientes quando trazem às consultas o relato de algum sonho, esperam que o terapeuta considere o que foi dito e que utilize esta informação em seu tratamento.

Alguns alcoolistas, na nossa experiência, conseguem aprender, a partir de seus sonhos, a identificar, não só o craving pelo álcool, como possíveis situações de risco de recaída. Um exemplo disto é o sujeito que, ao sonhar que está bebendo com um determinado amigo dependente ou em um local de risco, cria antecipadamente estratégias para poder evitar ou enfrentar estas situações de risco.

\section{CONCLUSÕES}

Uma das dificuldades ao elaborar esta revisão foi a carência de estudos abordando os sonhos dentro da abordagem Cognitivo-Comportamental, assim como o reduzido número de pesquisadores que têm acesso a um laboratório do sono com recursos para realizar pesquisas mais incrementadas do ponto de vista científico.

Percebe-se que o trabalho do pesquisador, neste sentido, é fundamental para que se forneçam subsídios para que os terapeutas sintam-se seguros para utilizar os sonhos como instrumento clínico, desfazendo a crença de que estes somente podem ser utilizados dentro da linha Psicanalítica.

No caso de dependentes químicos, é de conhecimento que os sonhos ainda são pouco utilizados pelos terapeutas, principalmente devido a muitos profissionais trabalharem com a Terapia Cognitivo-Comportamental. Este estudo não tem, portanto, a pretensão de esgotar este tema, mas sim de suscitar o interesse para que novas investigações sejam feitas, tendo por foco a análise dos sonhos, de acordo com esta abordagem terapêutica, e sua utilização na área da dependência química.

Os sonhos poderão, no futuro, ser melhor químicos, como é o caso dos alcoolistas, e utilizados como um importante instrumento terapêutico que virá em auxílio aos profissionais na difícil luta pela manutenção da abstinência.

\section{REFERÊNCIAS BIBLIOGRÁFICAS}

1. Edwards G, Marshall E Jane \& Cook, Chiristopher $\mathrm{CH}$. O tratamento do alcoolismo. Porto Alegre: Artes Médicas; 1999.

2. Oliveira M. Entrevista Motivacional. In Cunha, Jurema e Colaboradores. Psicodiagnóstico V. Porto Alegre: Artes Médicas; 2000. p. 88-95.

3. Skinner BF. Behaviorism at fifty. Science, 1963; 140: 951-958.

4. Guillardi JH. Um modelo comportamental de análise de sonhos. In Rangé, Bernard. Psicoterapia Comportamental e Cognitiva de Transtornos.Psiquiátricos. Campinas: Editorial Psy II; 1998. p. 257-267.

5. Bachtold L. Os Sonhos na Terapia Comportamental. Curitiba: InterAção - Revista do Departamento de Psicologia da UFPR, 1999; v. 3, 21-34.

6. Delitti M. Relato de sonhos: como utilizá-los na prática da Terapia Comportamental. In Wielenska RC et al. Sobre o comportamento e cognição-Questionando e Ampliando a teoria e as intervenções clínicas em outros contextos. Santo André, São Paulo: ARBytes, v. 6, 1. ${ }^{\mathrm{a}}$ ed. , 2000;. p. 204-210.

7. Martinez D. Como vai seu sono?_Porto Alegre: AGE; 2000.

8. Dixon M \& Hayes L. A Behavioral Analysis of Dreaming. The Psychological Record, 1999; 49, 613-628.

9. Reimão, Rubens. Sono - Estudo Abrangente. São Paulo: Atheneu; 1996.

10. Freud S. A Interpretação dos Sonhos. Obras Completas, Vol. IV. Rio de Janeiro: Imago; 1900/1987.

11. Chaves M, Caixeta M \& Machado D da C. Neuropsicologia da Atividade Onírica - Considerações teóricas sugeridas por aspectos clínicos. Arquivos de Neuropsiquiatria, 1997; 55,_3-B, 661-665.

12. Schredl M. Continuity Between Waking Life and Dreaming: Are All Waking Activities Reflected Equally Often in Dreams? Perceptual and Motor Skills; 2000, 90, 844-846.

13. Kalra R, Natu MV, Deswal RS \& Agarwal AK. Dream Features.in Psychiatric Patients on Multiple Psychoactive Drugs. Human Psychopharmacology Clinical Experimental., 2000 15, 525-528.

14. Colace C. Dreams in Abstinent Heroin Addicts: Four cases Reports. Sleep and Hypnosis; 2000, 2,_4, 160-163.

15. Usher R. O sono. São Paulo: Saraiva; 1991.

16. Drummond SP, Gillin JC, Smith TL \& DeModena A. The sleep of abstinent pure primary alcoholic patients: natural course and relationship to relapse. Alcoholism: Clinical and Experimental Research, 1998, 22,8, 1796-1802.

17. Schredl M. Dream Recall in Patients With Primary Alcoholism After Acute Withdrawal. Sleep and Hypnosis, 1999, 1, 1, 35-40.

18. Araujo RB. A relação entre sonhos e craving em alcoolistas na fase de desintoxicação. [ Dissertação de Mestrado]. Porto Alegre: PUCRS; 2002.

19. Weinstein A, Lingford, Hughes A, Martinez, Raga J \& Marshall J. What makes alcohol-dependent individuals early in abstinence crave for alcohol: exposure to the drink, images of drinking, or remembrance of drinks past? Bristol: Alcoholism: Clinical and Experiemental Research., 1998, 22, 6, 1376- 1381.

20. Choi SY. Dream as a prognostic factor in alcoholism. 
American Journal of Psychiatry, 1973, Vol. 130,6, 699702.

21. Christo G \& Franey C. Addicts' drug-related dreams: their frequency and relationship to six-month outcomes. Substance Use \& Misuse, 1996, 31, 1, 1-15.

22. Johnson RA. Relationship of dreams of drinking and negative expectancies to alcohol treatment outcome in a veterans administration alcoholic inpatient treatment population. Dissertation. Abstracts. International; Section $\mathrm{B}$, The Sciences and Engineering, 2000, Vol. 61 (6B): 3280 .

23. Peters KA. The dreams of alcoholic men in early sobriety. Dissertation Abstracts International, Section B, The Sciences and Engineering, 1997, Vol. 57, 10-B, 6588.

\section{RESUMO}

Este trabalho tem por objetivo realizar uma revisão teórica a respeito do sono e dos sonhos de alcoolistas de acordo com a Terapia Cognitivo-Comportamental, aprofundando aspectos cognitivos e neurológicos. Na medida em que esta é a abordagem psicoterápica mais indicada para dependência química, os sonhos, utilizados na Terapia Psicanalítica, tiveram de ser estudados sob o enfoque cognitivocomportamental, para que pudessem ser aproveitados na clínica com estes pacientes. As alterações no sono de alcoolistas, por exemplo, pode servir como alerta quanto a um risco de recaída, o que indica que a avaliação do sono não pode ser desconsiderada pelos profissionais, durante o tratamento destes pacientes. O relato dos sonhos, por sua vez, pode ser um instrumento terapêutico muito valioso por oferecer pistas a respeito do quanto o craving está presente naqueles que estão tentando manter-se em abstinência. Se os profissionais forem bem sucedidos quanto à identificação precoce de uma situação de risco como o craving, poderão ser mais efetivos quanto à prevenção da recaída. Conclui-se quanto à necessidade de que sejam feitas novas pesquisas a respeito dos sonhos de acordo com esta abordagem teórica, bem como avaliada a sua aplicação na área da dependência química.

Descritores: Álcool, sonho, sono, Cognitivo-Comportamental.

\section{ABSTRACT}

This work is aimed to carry out a theoretical review of sleep and dreams of alcoholics according to cognitive-behavioral therapy, and focusing on cognitive and neuroligical aspects. Considering that this approach is more indicated to chemical dependency, dreams, which are frequently used in Psychoanalytic Therapy, have to be studied under a cognitive-behavioral approach, so they could be used in clinics with these patients. The alterations in sleep of alcoholics, for example, could be taken as an alert about the risk of relapse, which indicate that sleep evaluation can not be disregarded by professionals, during the treatment of these patients. The reporting of dreams, by itself, can be a therapeutic instrument, very valuable at offering clues in respect of how much cravings are present in those who are trying to keep clean. If the professionals are successful about early identification of a risk situation as a craving, they could be more effective about the prevention of relapse. We concluded about the necessity of new researches on dreams according to this theoretical approach, as well as to evaluate their application in the chemical dependency field.

Keywords: Alcohol, sleep, dream, Cognitive-Behavioral therapy.

Title: Dreams of Alcoholics According to CognitiveBehavioural Therapy

\section{RESUMEN}

Este trabajo tiene como objetivo realizar uma revisión teórica a respecto del sueño y de los sueños de alcohólicos, teniendo en vista la Terapia Cognitivo-Comportamental, profundizando aspectos cognitivos y neurológicos. Em la medida en que éste es el abordaje psicoterápico más indicado para dependencia química, los sueños, utilizados en la Terapia psicoanalítica, tuvieron que ser estudiados sobre el enfoque cognitivo-comportamental, para que pudieran ser aprovechados en la clínica con estos pacientes. Las alteraciones em el sueño de los alcohólicos, por exemplo, pueden servir como alerta para el riesgo de recaída, lo que indica que una evaluación del sueño no puede ser desconsiderada por los profesionales durante el tratamiento de estos pacientes. El relato de los sueños, a su vez, puede ser un instrumento terapéutico muy valioso por ofrecer pistas a respecto de cuanto el "craving" existe en aquellos que están intentando mantenerse em abstinencia. Identificando situaciones de riesgo como el "craving", los profesionales podrían ser más efectivos en la prevención de la recaída.

Se concluye que existe la necesidad de realizar nuevos estudios teóricos a respecto de los sueños con este mismo abordaje teórico, así como evaluar su aplicación en el área de la dependencia química.

Palabras-clave: Alcohol, sueños, sueño, CognitivoComportamental.

Título: El abordage cognitivo-comportamental de los sueños de alcoholicos

Endereço para correspondência:

Pontifícia Universidade Católica do

Rio Grande do Sul

Av. Ipiranga, 6681, Caixa Postal 1429 - Prédio 11

90616-900 - Porto Alegre - RS

E-mail: renataudbrasil@hotmail.com

Copyright (c) Revista de Psiquiatria

do Rio Grande do Sul - SPRS 